findet noch zu selten ein Lernen durch Training, zum Beispiel durch Simulationstraining, statt. Interessanterweise ist längst belegt, dass Teamleitungs- und Kommunikationstraining einen höheren Einfluss auf den CPR-Erfolg haben als ein reines Algorithmentraining. In genau diesem Kontext ist die positive Aussage der Studie von Thomas et al. zu sehen: Die moralische Belastbarkeit steigt und im Gegenzug sinkt die moralische Not jedes einzelnen CPR-Teammitglieds, wenn die Qualität der Teamarbeit auf Grundlage eines Trainings, wie etwa durch "Crisis-Resource-Management"(CRM)Training, verbessert wird.

Ein CRM-Training in der Realität im Arbeitsalltag umzusetzen, bereitet zweifellos zahlreiche Probleme. Neben Schwierigkeiten mit dem technischen Equipment und vor allem zeitlichen Einschränkungen auf Seiten der Kursteilnehmer wie auch der Instruktoren existieren typische „Widerstände" im Bereich Teamzusammensetzung, Erwartungen und Ängste und auf Ebene der Teamkommunikation. Aufgezählt seien an dieser Stelle Aspekte wie fehlende Bereitschaft Einzelner (sog. „freie Künstler"), überhaupt teilzunehmen, Festhalten an
Gewohnheiten („Das haben wir noch nie gemacht", "Was soll das Ganze denn überhaupt?" oder "Es läuft doch alles perfekt bei uns!") und fehlende Ernsthaftigkeit gegenüber der Simulation („Puppenspielerei“). Außerdem kommen voreingenommen-fatalistische und somit unverantwortliche Ablehnung gegenüber dem Trainingsgedanken als gefahrlose und den Ernstfall vorbereitende Trockenübung hinzu („Ich lerne viel besser an meinen Fehlern am Patienten; die prägen sich mir einfach besser ein!"), Ängste vor Machtverlust, gering entwickelte Kritikkultur, stattdessen Annahme einer anscheinend sicheren Hierarchie und beruhigenden Harmonie.

Positive Änderungen vollziehen sich auch hier nur in kleinen Schritten. Aber sie finden statt. Und wissenschaftliche Arbeiten wie die von Thomas und Kollegen untermauern die Sinnhaftigkeit all dieser Bemühungen. Erfreulicherweise unterstützen die Fachgesellschaften und die Politik längst diese Sichtweise.

Die Kernaussage der Autoren wurde im Editorial der gleichen Ausgabe der Pediatric Critical Care Medicine von Morrison und Berg bestätigt und hervorgehoben, der

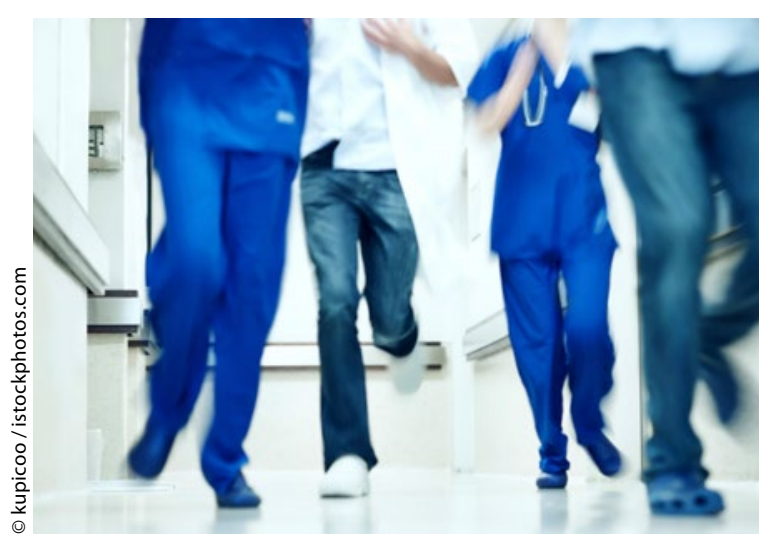

Steht eine kardiopulmonale Reanimation an, dann ist das gesamte Team idealerweise durch regelmäßiges Training gerüstet.

meines Erachtens nichts hinzuzufügen ist, deswegen nachfolgend im Original: „Moral excellence results from making the protection and promotion of the patient's life and health one's primary concern, keeping self-interest secondary. Making and sustaining these integrity-related commitments establish one's identity as a professional clinician."

Dr. Thomas Hoppen

\title{
Entzündungen im Windelbereich vermeiden
}

\section{Eine retrospektive Studie aus der Türkei untersuchte 63 Säuglinge im Hinblick auf eine Windeldermatitis. Sie stellten fest, dass Feuchttücher eher begünsti- gend sind, Stillen dagegen eine schützende Wirkung zeigt.}

D ie Kinder der Untersuchung hatten im Median ein Alter von rund 11 Monaten, circa $40 \%$ waren jünger als sechs Monate. Schwere Formen von Windeldermatitis zeigten sich bei circa $22 \%$, moderate bei rund $58 \%$ und leichte Formen bei $20 \%$ der Kinder. Drei Viertel der Probanden waren gestillt, ein Drittel davon komplett. Anamnestisch hatten gestillte Kinder in der Vergangenheit signifikant seltener eine Windeldermatitis (durchschnittlich eine gegenüber zwei bei nicht gestillten Kindern). Bei circa $75 \%$ der Kinder konnte eine Candida-Infektion nachgewiesen werden, Säuglinge mit Windelsoor hatten häufiger ein Rezidiv mit bereits einer schon vorangegangenen ersten Pilzinfektion. Augenscheinlich förderte auch die Säu- berung der Windelregion mit Feuchttüchern im Vergleich zu Wasser und Waschlappen aus Baumwolle die Entwicklung einer Candida-Dermatitis

Therapeutisch konnte bei knapp $60 \%$ der Kinder innerhalb von 3-5 Tagen eine Besserung erzielt werden, bei etwas über $80 \%$ innerhalb von zehn Tagen eine weitgehende Abheilung. Hauptsächlich wurden Schutzpasten und bei nachgewiesenen Candida-Infektionen zusätzlich topische Antimykotika über 10-14 Tage verwendet, bei ausgeprägten Dermatitiden auch kurzfristig niedrigpotente topische Kortikosteroide wie zum Beispiel 0,125\%ige Prednisolon-Creme über 3 Tage.

Die Autoren aus Ankara empfehlen außerdem, bei Neugeborenen bis zu zwölfmal, später im ersten Lebensjahr acht- bis sechsmal pro Tag eine neue Windel anzulegen, möglichst solche mit höherer Saugkraft. Zur Prophylaxe einer Windeldermatitis raten sie zusätzlich zu einer Hautschutzcreme. Große Bedeutung messen sie auch dem Stillen bei, da der Stuhl von gestillten Kindern einerseits einen niedrigeren $\mathrm{pH}$-Wert und andererseits geringere Aktivitäten von Proteasen und Lipasen hat, weshalb es zu weniger Irritationen der Haut im Windelbereich kommt.

Ersoy-Evans S et al. Diaper Dermatitis: A Review of 63 Children. Pediatr Dermatol 2016;33:332-6

\section{Kommentar}

Bei einer Windeldermatitis sind Superinfektionen mit Hefepilzen der Gattung Candida sehr häufig. Präventiv sind längeres Stillen und die Vermeidung von Feuchttüchern zu empfehlen.

Dr. Ulrich Mutschler 